\title{
Fine Needle Aspiration Cytology Verses Trucut Biopsy In Preoperative Planning Of Surgical Treatment Of Breast Lumps
}

\author{
Dr R K Shastri, MS (General Surgery) \\ Associate Professor of Surgery Dr Pinnamaneni Siddharth Institute of Medical sciences and Research \\ Foundation Chinaoutpalli, Krishna District, Andhra Pradesh, India
}

\begin{abstract}
Brest lumps are frequently found in females of different age group. So a need arises to distinguish benign and malignant lesions, prior to taking a decision regarding a definitive treatment. Increase awareness and better understanding of the problem has resulted in a marked increase in number of tumors detected. Breast lumps are detected by SBE by the patients, next a clinical breast examination by surgeon and then a breast imaging. To arrive at an accurate diagnosis a cytological analysis is needed. So an easy to perform technique like a FNAC or Trucut biopsy can be carried out which is cost effective and acceptable to the patients. It can be done in a busy out patient clinic without much preparation or expensive equipments. An excisional biopsy with a safe margin is done in case of a small lump. Combinations of various approaches tried to increase specificity and sensitivity for a proper diagnosis. Aspiration cytology was thought to be of great value provided it is positive for malignant cells. But a negative FNAC can not for sure rule out a malignancy. Though relatively easily performed, its not reliable for a presurgical decision making and adequate management of breast lump cases. Trucut biopsy can provide all necessary details to guide the surgeon to design an appropriate therapeutic strategy.
\end{abstract}

Keywords: Fine needle aspiration cytology, Trucut biopsy, automated core biopsy device

\section{Introduction}

Trucut biopsy is a safe method for a histological diagnosis, in operable breast cancer cases and a reliable technique to assess hormone receptor status.

Aim- To find out the diagnostic accuracy of FNAC and trucut biopsy as compared to the final histopathological report in breast lumps.To compare diagnostic accuracy of FNAC and trucut biopsy differentiating the benign and malignant lesions of palpable breast lumps. Also to analyse the sensitivity, specificity, positive and negative predictive values and the efficacy of FNAC and trucut biopsy with histopathological correlation.

\section{Materials and Methods}

This is a prospective study of 39 consecutive cases between October 2013 to June 2016. Females between age of 15 to 70 years with palpable lumps of variable duration were included. Frank advanced lesions, or lesions with skin infiltration were excluded from the study. Fine needle aspiration cytology was done for all of them. Those who could be motivated for a trucut biopsy subsequently, were only included in the study.The FNAC report, Trucut biopsy report and the final histopathology report of the excised specimen were compared. FNAC- Aspiration cytology obtained by a $21 / 22$ gauge needle and $10 \mathrm{ml}$ syringe, with multiple passes through the lump with negative pressure in the syringe. Aspirated material is spread on a glass slide, dried and fixed with 95\% alcohol, stained with hematoxylin and eosin before being studied by a cytopathologist.

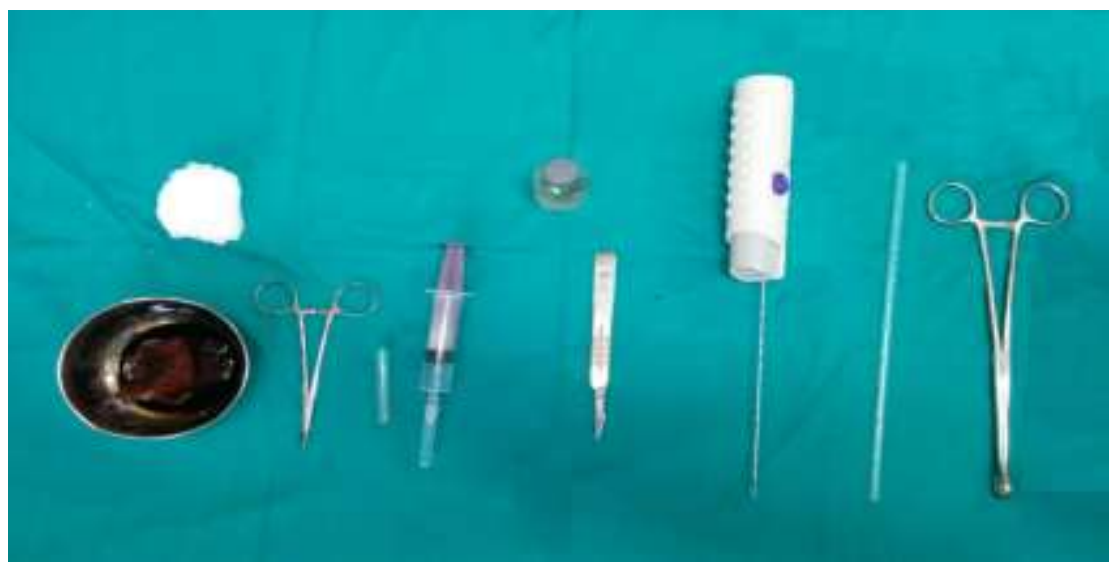

Equipments for a trucut biopsy 
Trucut biopsy procedure is similar to FNAC, except a wide bore needle (11-14 gauge) is used and done under local anaesthesia, being infiltrated at the site of needle insertion. A nick is made in the skin with a no. 11 blade for insertion of the needle by an automated device. At least 4 bits of tissue obtained from a breast lump ensuring an adequate sampling and each time needle inserted in different directions. Tissue specimens are put in $10 \%$ buffered formalin in vials, to be processed to paraffin blocks[5]. For vaguely palpable lumps and small lumps at a depth, imaging assists to guide the needle to the desired tissue. Normally a free hand technique is good enough to obtain adequate tumor tissue. Margins of the tiny cut is approximated with a strip of micropore.

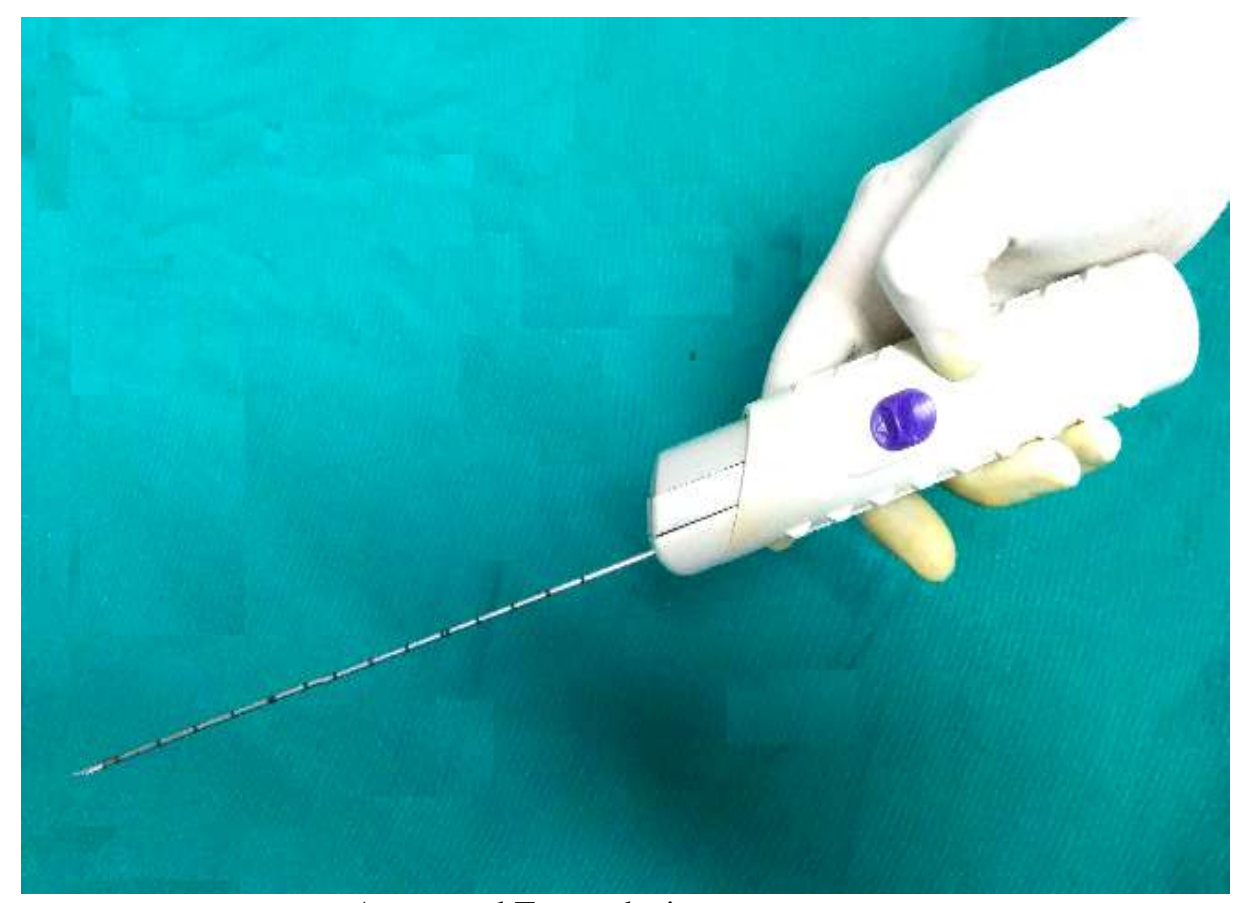

Automated Trucut device

Observation and result- Most common age group having breast lump was 25-34 years. The incidence of malignant lesions were found mostly in 45-70 years age group.

Distribution of patients
according to their age
\begin{tabular}{|c|c|c|}
\hline Age group & Total \\
\hline & Frequency & Percentage \\
\hline $15-24$ & 7 & 17.9 \\
\hline $25-34$ & 11 & 28.2 \\
\hline $35-44$ & 8 & 20.5 \\
\hline $45-54$ & 7 & 17.9 \\
\hline $55-64$ & 5 & 12.8 \\
\hline 765 & 1 & 2.6 \\
\hline Total & 39 & $100 \%$ \\
\hline
\end{tabular}

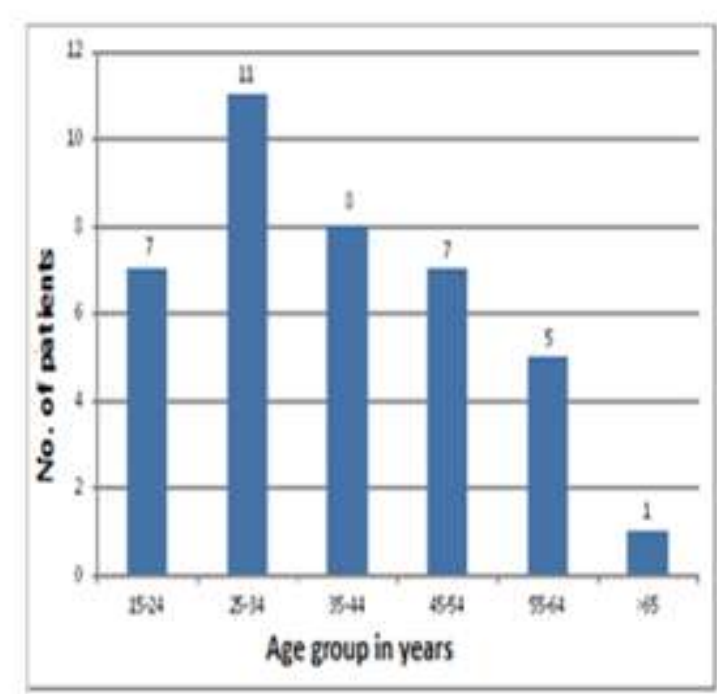

The size of beast lumps ranged from 2-12 cms. $92 \%$ of benign lesions were up to $5 \mathrm{~cm}$ size. Malignant lesions ranged beween 5 to $11.8 \mathrm{~cm}$ and among them71.42\% measured $5-10 \mathrm{~cm}$ in diameter. In addition to breast lump 2 cases had pain in lump, one had bloody discharge from nipple and 3 cases had axillary nodes. 


\begin{tabular}{cccccc}
\hline $\begin{array}{c}\text { Size of lump } \\
\text { In cm. }\end{array}$ & Benign & Percentage & Malignant & Percentage & Total \\
\hline$<5$ & 23 & 92 & 2 & 14.29 & 25 \\
\hline $5-10$ & 2 & 8 & 10 & 71.42 & 12 \\
$>10$ & 0 & 0 & 2 & 14.29 & 2 \\
\hline Total & 25 & 100 & 14 & 100 & 39 \\
\hline
\end{tabular}

Distribution of patients according to size of lump

Out of the 18 patients 2 had the family history of cancer breast, in their mothers. Both of them suffered from infiltrating duct carcinoma.

\begin{tabular}{|c|c|c|c|c|}
\hline & \multicolumn{3}{|c|}{ BENIGN } & \multicolumn{2}{c|}{ MALIGNANT } \\
\hline $15-24$ & FREQUENCY & PERCENTAGE & FREQUENCY & PERCENTAGE \\
\hline $25-34$ & 9 & 33.33 & 0 & 0 \\
\hline $35-44$ & 4 & 42.86 & 2 & 11.11 \\
\hline $45-54$ & 1 & 4.76 & 6 & 22.22 \\
\hline $55-64$ & 0 & 0 & 5 & 27.78 \\
\hline$>65$ & 0 & 0 & 1 & 5.56 \\
\hline TOTAL & 21 & 100 & 18 & 100 \\
\hline
\end{tabular}

Age wise distribution of patients having benign and malignant lesions

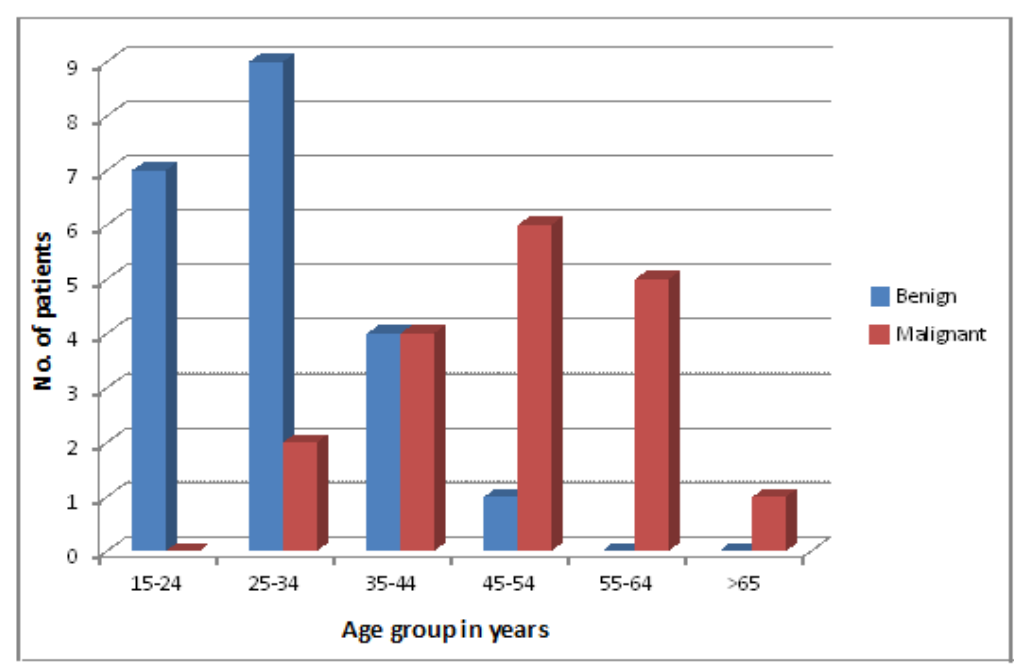

Out of 25 cases of benign FNAC report, 21 cases were confirmed by histopathology. False negative were 5 cases. False positive was zero. One case was reported as nonconclusive. On trucut biopsy as well as histopathology of excised specimen later confirmed the diagnosis. 


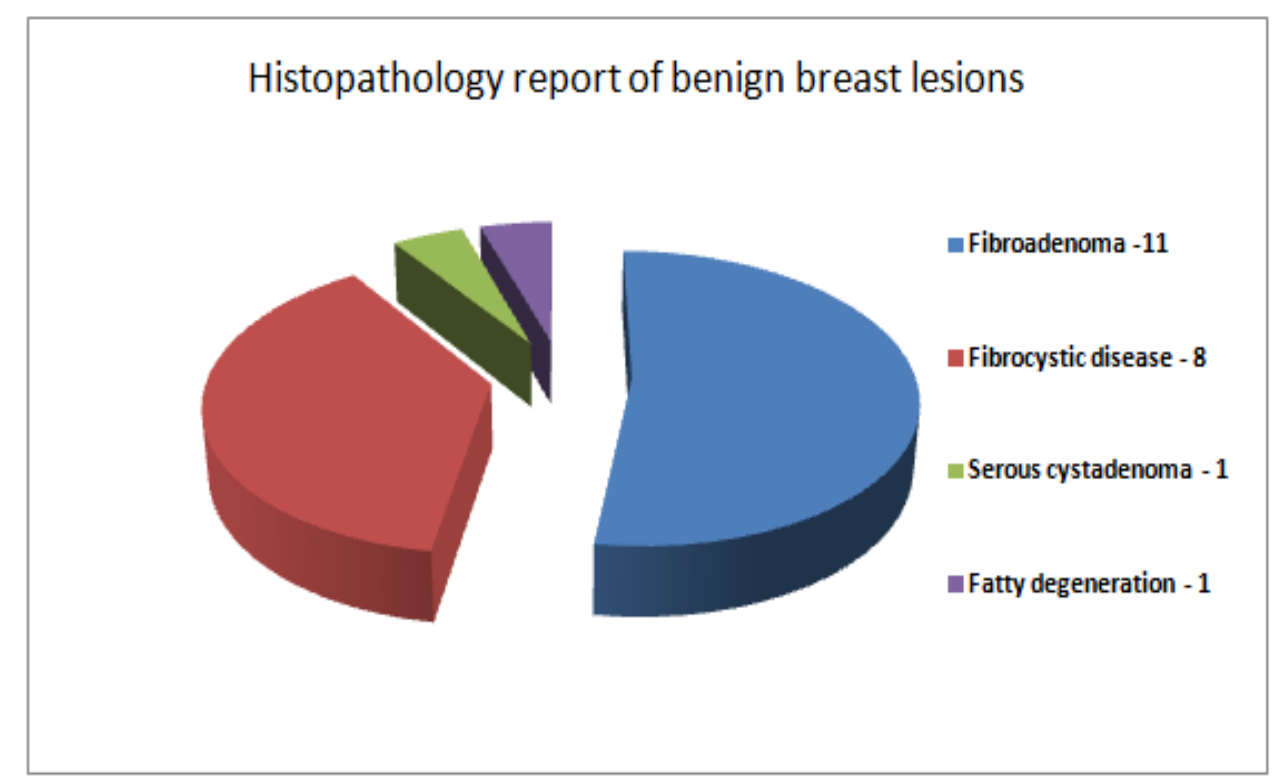

Accuracy of a test is determined by sensitivity and specificity. Sensitivity refers to the proportion of the times that a test yields true positives. If it is close to $100 \%$ it is more likely to be the correct diagnosis. Specificity refers to the proportion of the time that a test yields true negatives. If it is close to $100 \%$ it is more likely to be a negative result means patient is truly free from the disease. If done perfectly a test gives only true positives and true negatives.

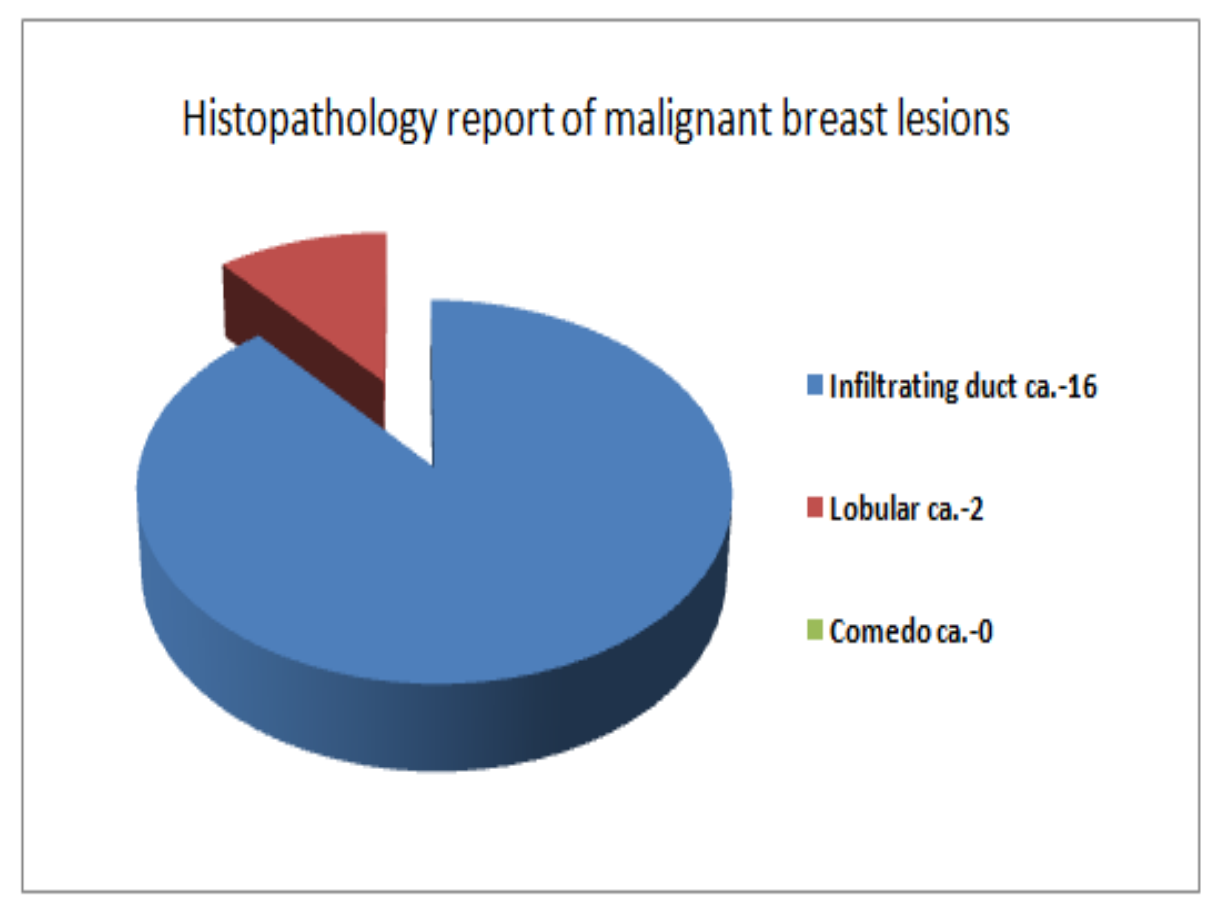

Any uncertainity makes the situation difficult for the surgeon to take a preoperative decision. If result is a false positive and a second test is mandatory to avoid an unnecessary surgical procedure. If the test is false negative and specimen histology proves it to be a malignant lesion, a second surgery may be needed for the patient.No medical test is perfect. False positive or false negative results are due to limitations of technology, human error or machine error. Any of these leads to reduced specificity and / or sensitivity for a test. 


\begin{tabular}{|c|c|c|c|}
\hline Benign & Malignant & $\begin{array}{l}\text { Non- } \\
\text { conclusive }\end{array}$ & Total \\
\hline 25 & 13 & 1 & 39 \\
\hline
\end{tabular}

Result of FNAC

\begin{tabular}{|c|c|c|}
\hline Benign & Malignant & Total \\
\hline 21 & 18 & 39 \\
\hline
\end{tabular}

Result of Trucut biopsy

\begin{tabular}{|r|c|c|}
\hline Benign & Malignant & Total \\
\cline { 2 - 3 } & 18 & 39 \\
\hline
\end{tabular}

Histopathology of excised lump

In this study the sensitivity of trucut biopsy is $95.45 \%$ and the specificity is $100 \%$. The positive and negative predictive values are $100 \%$ each. No inadequate sampling was reported for trucut biopsy. So the accuracy rate of the needle tip localizing the tumor in trucut biopsy was $100 \%$.

Predictive value of FNAC

\begin{tabular}{|l|l|l|}
\hline Positive & True positive- 13 & False positive -0 \\
\hline Negative & True negative-20 & False negative - 5 \\
\hline
\end{tabular}

Predictive value of Tru cut biopsy

\begin{tabular}{|l|l|l|}
\hline Positive & True positive- 21 & False positive - 0 \\
\hline Negative & True negative- 17 & False negative - 1 \\
& & \\
\hline
\end{tabular}

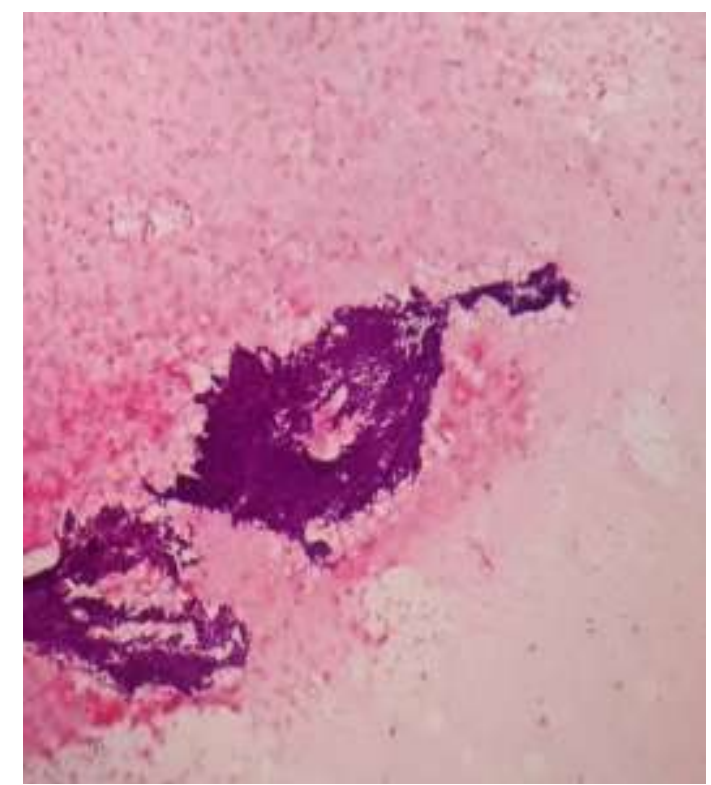

FNAC- Benign proliferaive breast disease

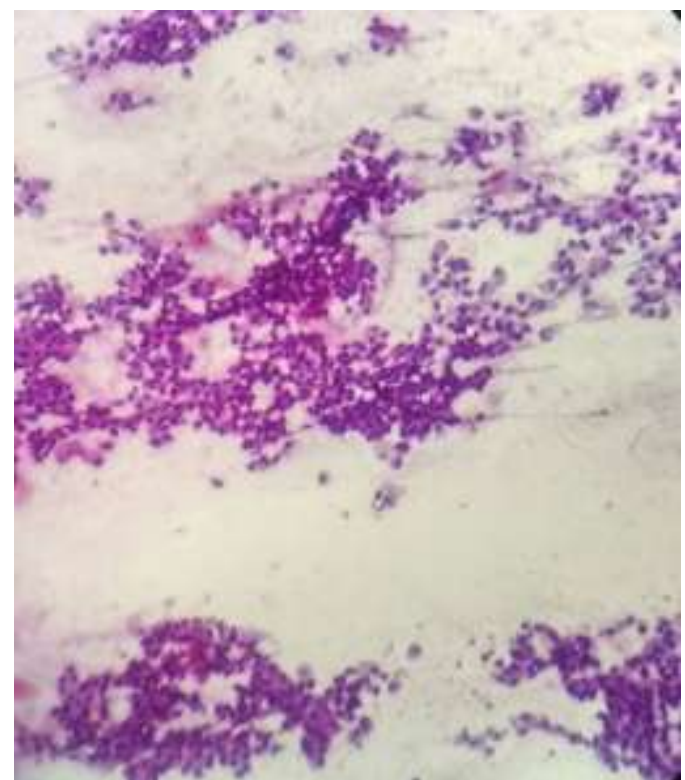

FNAC- suggests malignant lesion of breast 


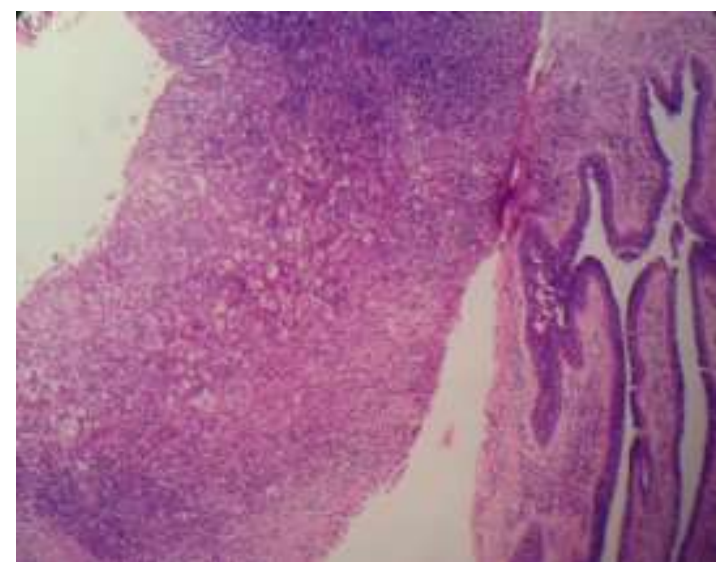

Trucut- Chronic nonspecific mastitis

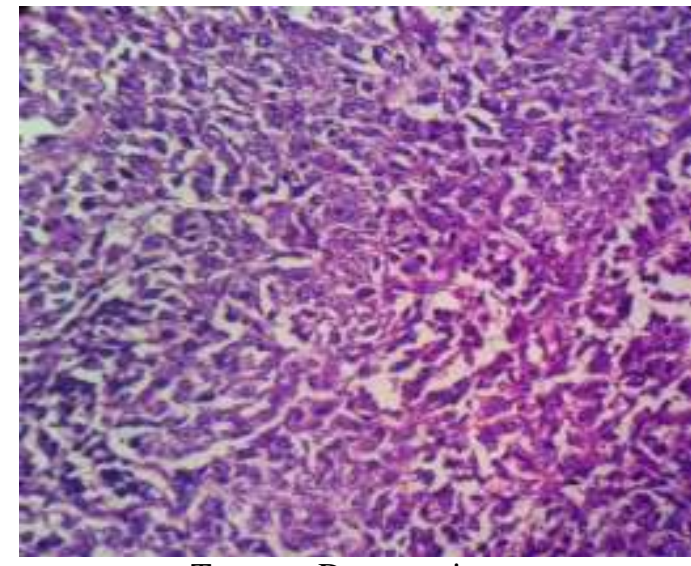

Trucut - Duct carcinoma

\section{Discussion}

Breast cancer is the most common female malignancy worldwide[1]. With an early detection and proper management the outcome is the best. In breast lump work up, the triple assessment protocol is followed, which includes- a clinical breast examination, imaging in form of mammogram / sonogram, or mammosonogram and a FNAC / Trucut biopsy. Though FNAC technique is simple, patients dislike repeated needling of the breast lump without anesthesia[2]. Trucut biopsy is rather easily accepted when patients are counselled well, regarding gravity of the situation and convinced of the lump being suspicious of a malignancy.

Even though a high degree of diagnostic accuracy of FNAC in breast cancer has been claimed in several studies, its role in management of breast lumps is still controversial. It is often non-conclusive and not the most suitable tool to be used in the diagnosis of breast lumps. Trucut needle biopsy is a good alternative. Also it is an alternative to open biopsy for neoplastic breast lesions[3,4]. Tissue samples should not be replaced by cytological material when core biopsies can be performed easily and without contraindications. In fact trucut biopsy can substitute both FNAC and open biopsy[4].

In spite of extensive use, the cytological findings of FNAC smears cannot be fully relied upon to take a decision to perform a definitive surgery. Core biopsy, based on study of architectural and cytological pattern of the obtained tissue specimens can clinch a diagnosis, even can find out an invasive cancer, if present. Thus a more expensive intra operative frozen section biopsy procedures can also be avoided. Core biopsy supports a definitive diagnosis, permits to take an immediate decision regarding treatment in $90 \%$ of cases. In case of diagnostic discordance between mammography and trucut biopsy, an intra operative frozen section biopsy used for decision making[4]. In trucut biopsy complication rates are low, a scar is avoided and its cost effective. When a breast lump is clinically and mammographically suspicious, the sensitivity and specificity of trucut biopsy is almost $100 \%$. False negative rates is extremely low[5]. Morbidity in terms of pain and bleeding is minimal in experienced hands[6].

Limited sample size obtained in trucut biopsy requires proper interpretation of the tissue histopathology. With definitive benign findings, only routine screening follow ups done without any need for further investigations. If malignancy detected, receptor status also assessed for a definitive treatment[7].

Trucut biopsy furnishes ample material for a precise diagnosis of the histological tumor type, its aggressiveness, grade, lympho vascular invasion, and receptors status. Thus it provides enough information useful to the surgical decision making process. The results can be correlated with final histopathology report. It also permits the eventual use of neo adjuvant therapy[8,9]. Image guided core needle biopsy is preferred for nonpalpable mammographic abnormalities and also considered as a minimally invasive diagnostic method for small palpable lesions that can be imaged[7,10,11].

In the present study, the breast lumps in 39 cases detected by clinical examination and imaging were subjected to FNAC, core biopsy and matched with final biopsy report of the surgical specimen. The sensitivity of core biopsy was $95.45 \%$, specificity and predictive value of positive result were $100 \%$. This study confirms that core biopsy should be routinely performed for preoperative diagnosis of breast lesions being a cost effective, safe and simple procedure to be performed in the office settings under local anaesthetics and without much preprocedure preparations[4]. FNAC to evaluate a breast lump shows a high sensitivity, specificity and accuracy $[6,12,13,14,15]$ but trucut always provides a better histological diagnosis and is more accurate and more trusted. Sampled cores of tissue obtained by a simple technique with an automated biopsy gun, can provide a more accurate answer to plan out specific surgical treatment of breast lumps. It can avoid multiple surgeries like an excision biopsy and next a definitive surgery. 


\section{Referrences}

[1]. Homesh N A, Issa M Aet al The diagnostic accuracy of fine needle aspiration cytology versus core needle biopsy for palpable breast lump(s).Saudi Med J 2005; 26(1): 42-46

[2]. Eman Muhammad et al, Validity of fine needle aspiration cytology (FNAC) in Diagnosis of breast lumps in upper Egypt, J. Med. Lab. Sci., Mar. 2012; 21(1):69-81

[3]. Mohamed T Musa M I, Randa Z A Khair et al., Diagnosis of Breast Cancer: is there any protocol which is applicable worldwide?Sudan JMS,June 2009,vol.4 issue2:157-162

[4]. M.L. CARUSO, G. GABRIELI et al, Core Biopsy as Alternative to Fine-Needle Aspiration Biopsy in Diagnosis of Breast Tumors, The Oncologist 1998;3:45-49

[5]. Schwartz's principles of Surgery: $9^{\text {th }}$ Edition 2010, pg 450

[6]. W S Yong, K H Chia et al. A Comparison of Trucut Biopsy with Fine Needle Aspiration Cytology in the Diagnosis of Breast Cancer, Singapore Med J 1999; Vol 40(09):

[7]. Sabiston text book of Srgery $18^{\text {th }}$ Edition Vol.1 2008 pg 860-861

[8]. Florentine BD, Cobb CJ, Frankel K et al. Core needlebiopsy: a useful adjunct to fine-needle aspiration in selectpatients with palpable breast cancer. Cancer Pathol 1997;81:33-39.

[9]. Mohammed Bdour, Saleh Hourani et al, Comparison between Fine needle aspiration cytology and tru-cut biopsy In the diagnosis of breast cancer, Journal of Surgery Pakistan (International) 13 (1) January - March 2008

[10]. Vic Velanovich, MD, Frank R. Lewis et al, Comparison of Mammographically Guided Breast Biopsy Techniques, Ann Surg. 1999 May; 229(5): 625.

[11]. Baily and Love's Short Practice Of Surgery $26^{\text {th }}$ Ed. 2013 Pg.800

[12]. Rahman MZ, et al,Fine Needle Aspiration Cytology of Palpable Breast Lump: Feb. 2013, A Study of 1778 Cases, Surgery: Current Research,

[13]. Khatun $\mathrm{H}$ et al (2002) Correlation of fine needle aspiration cytology and its histopathology in diagnosis of breast lumps.Bangladesh Med Res Counc Bull 28:77-81.

[14]. Kaminsky DB (1981) Aspiration biopsy of the community hospital. Masson Pub, USA.

[15]. Russ JE, Winchester DP, Scanlon EF, Christ MA (1978) Cytologic findings of aspiration of tumors of the breast. Surg Gynecol Obstet 146: 407-411 